\title{
Refractory Seborrheic Dermatitis of the Head in a Patient with Malignant Lymphoma
}

\author{
Kayo Okada Yuichiro Endo Akihiro Fujisawa Miki Tanioka \\ Kenji Kabashima Yoshiki Miyachi \\ Department of Dermatology, Graduate School of Medicine, Kyoto University, Kyoto, Japan
}

\section{Key Words}

Seborrheic dermatitis · Paraneoplastic syndrome · Peripheral T cell lymphoma

\begin{abstract}
Seborrheic dermatitis is an inflammatory eruption that tends to distribute on the sebaceous areas of the body and is rarely described as a paraneoplasia. Here we report a case with a responsive seborrheic dermatitis-like eruption of the head which resulted in generalized erythroderma. Intensive examinations detected concurrent malignant lymphoma.
\end{abstract}

(c) 2014 S. Karger AG, Basel

\section{Case Report}

An 81-year-old woman recognized pruritic scaly erythemas on the scalp 2 months before the visit to our hospital. Her medical history included nephrosis syndrome that had been treated with oral cyclosporine $100 \mathrm{mg} /$ day for 8 years. The results of routine blood work before the onset of skin eruptions had been normal. Although the head erythemas appeared to be seborrheic dermatitis (SD) [1], the lesions did not respond to topical steroidal lotion. When she visited our hospital, the eruption had disseminated to the trunk and extremities, resulting in generalized erythroderma (fig. 1). Laboratory tests showed white blood cells 13,900/ $\mu$ l (normal 3,500-9,800), hemoglobin $11.9 \mathrm{mg} / \mathrm{dl}$ (normal 11.315.5), platelets 271,000/ $\mu \mathrm{l}$ (normal 155,000-365,000), eosinophils $19.0 \%$, atypical lymphocytes $2.0 \%$, lactate dehydrogenase $517 \mathrm{IU} / \mathrm{l}$ (normal 176-353) and soluble interleukin-2 receptor $1,510 \mathrm{U} / \mathrm{ml}$ (normal $<550$ ); human T-lymphotropic virus type I antibody was negative. A skin biopsy of the erythema of the trunk showed that lymphocytes 
Okada et al.: Refractory Seborrheic Dermatitis of the Head in a Patient with Malignant Lymphoma

and eosinophils had infiltrated dominantly to the superficial perivascular space. There was no superficial dermal infiltrate of lymphocytes. A biopsy of her scalp lesion indicated only spongiotic dermatitis with parakeratosis. There was no atypical folliculotropic cell infiltration around follicles (fig. 2). Polymerase chain reaction and flow cytometry of the skin specimens failed to detect malignant cells. However, atypical lymphocytes (basket cells) were detected in the peripheral blood and bone marrow (fig. 3). Flow cytometry of the blood and bone marrow specimens showed that malignant cells were positive for CD2, CD3, CD4, CD5, CD7, CD25 and CD45RA. Positron emission tomography-computed tomography revealed abnormal enhancement of axillary and inguinal lymph nodes and bone marrow. We consulted a hematologist and peripheral $\mathrm{T}$ cell lymphoma not otherwise specified (PTCLNOS), stage IV according to Ann Arbor [2] was diagnosed. PTCL can cause SD-like dermatitis that gradually develops into auto-sensitization dermatitis. As chemotherapy was dismissed considering her age, we administered fexofenadine $120 \mathrm{mg} /$ day and oral prednisolone 20 $\mathrm{mg} /$ day so as to alleviate erythroderma and pruritus. Although most of the eruptions improved within a week, pruritus persisted. We added pregabalin $50 \mathrm{mg} /$ day according to the recommendation by Yosipovitch [3], which reduced pruritus dramatically. The amount of daily oral steroid was tapered to $5 \mathrm{mg}$ over 4 months, maintaining remission of cutaneous symptoms.

\section{Discussion}

Few publications have described SD as a paraneoplastic syndrome in cases with lung cancer and lymphoma [4-6]. The diagnosis of paraneoplastic syndrome usually requires concurrent onset and parallel course with malignancy at least. Although we could not confirm whether the severity of the SD would parallel that of PTCL, SD seems to be a paraneoplasia since the onset of the SD coincided with that of PTCL and the SD was resistant to ordinary topical therapy.

Hematologic malignancies are often accompanied by severe pruritus. For chronic paraneoplastic pruritus, antidepressant and anticonvulsive drugs such as gabapentin are anecdotally effective [3]. Although the mechanisms of pruritus as well as the antipruritic effect of anticonvulsive agents are not clear, they probably inhibit central itch pathways. We used low-dose pregabalin that worked and accelerated the improvement of SD. This may be an important option for severe paraneoplastic pruritus.

In this case, simple SD and subsequent auto-sensitization dermatitis were suspected at first. However, the SD was too resistant to ordinal therapy and developed to erythroderma. Complete blood count revealed the presence of lymphoma that became an important clue to diagnosis. The differential diagnosis was folliculotropic mycosis fungoides. However, this possibility was ruled out by the results of pathological examinations.

\section{Disclosure Statement}

The authors report no conflicts of interest. There were no funding sources.

\section{References}

1 Burns T, Breathnach S, Cox N, Griffiths C (eds): Rook's Textbook of Dermatology, ed 8. Oxford, Blackwell, 2010, pp 23.29-23.34. 
Okada et al.: Refractory Seborrheic Dermatitis of the Head in a Patient with Malignant Lymphoma

2 Lister TA, Crowther D, Sutcliffe SB, Glatstein E, Canellos GP, Young RC, Rosenberg SA, Coltman CA, Tubiana M: Report of a committee convened to discuss the evaluation and staging of patients with Hodgkin's disease: Cotswolds meeting. J Clin Oncol 1989;7:1630-1636.

3 Yosipovitch G: Chronic pruritus: a paraneoplastic sign. Dermatol Ther 2010;23:590-596.

4 Kokturk A, Ikizoglu G, Kaya TI, Baz K, Ulubas B, Apa DD: Seborrhoeic dermatitis-like manifestation of lung cancer evolving into erythrodermia. J Eur Acad Dermatol Venereol 2004;18:381-382.

5 Venkateswaran S, Garg BR, Reddy BS, Narasimhan R: Cutaneous lymphoma mimicking seborrhoeic dermatitis. Indian J Dermatol Venereol Leprol 1995;61:45-47.

6 Bittencourt AL, de Oliveira Mde F: Cutaneous manifestations associated with HTLV-1 infection. Int J Dermatol 2010;49:1099-1110.
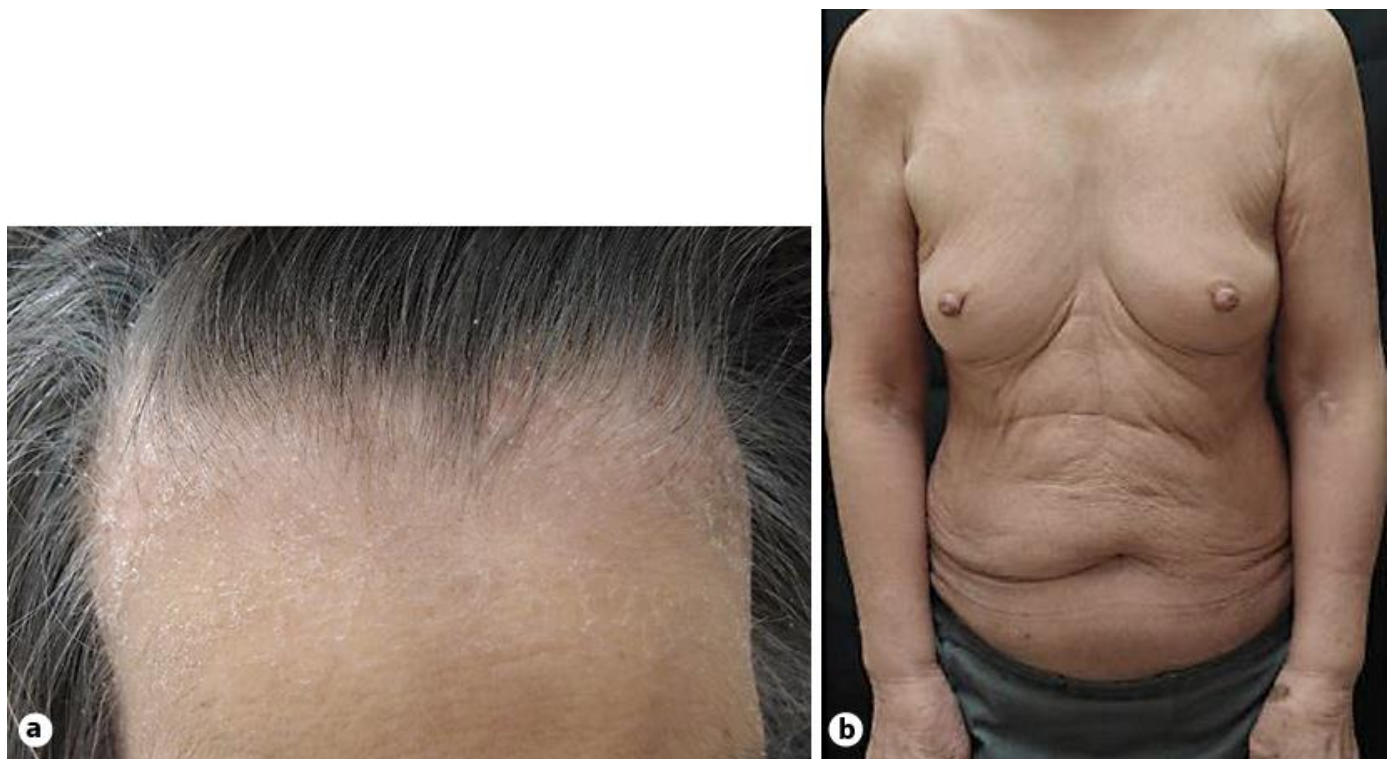

Fig. 1. a Scaly erythemas on the patient's head. b Generalized erythroderma of the trunk and extremities. 


\section{Case Reports in Dermatology}

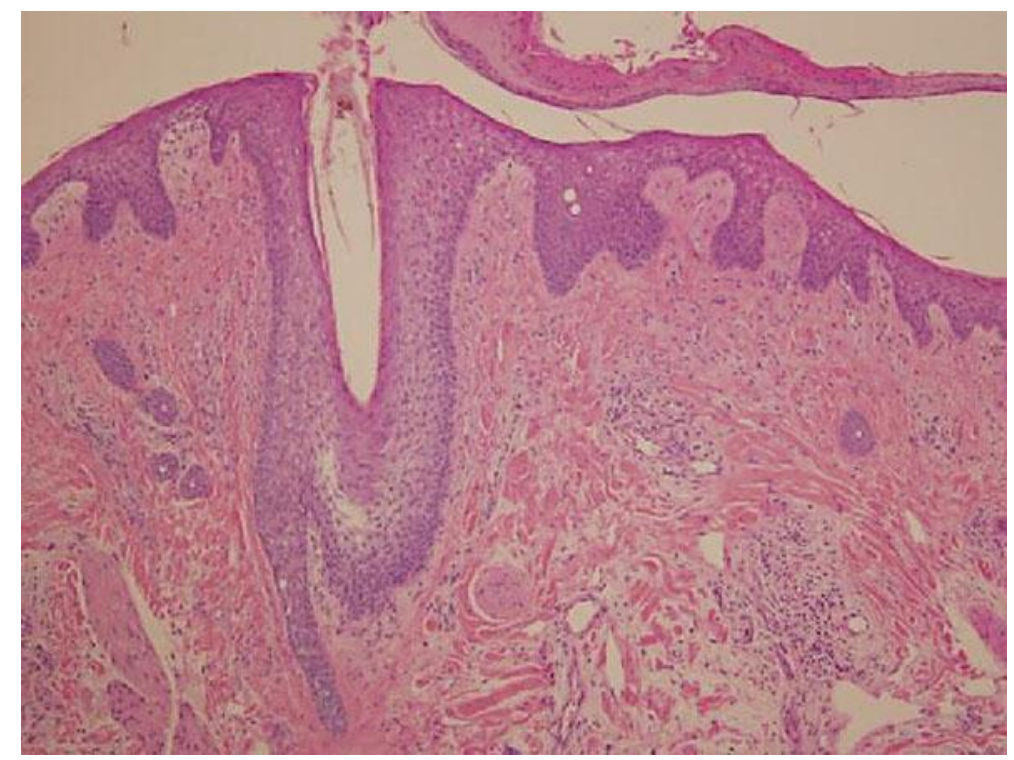

Fig. 2. There was no atypical folliculotropic cell infiltration around follicles. The biopsy of the scalp lesion indicated only spongiotic dermatitis with parakeratosis. Hematoxylin and eosin stain, $\times 100$.

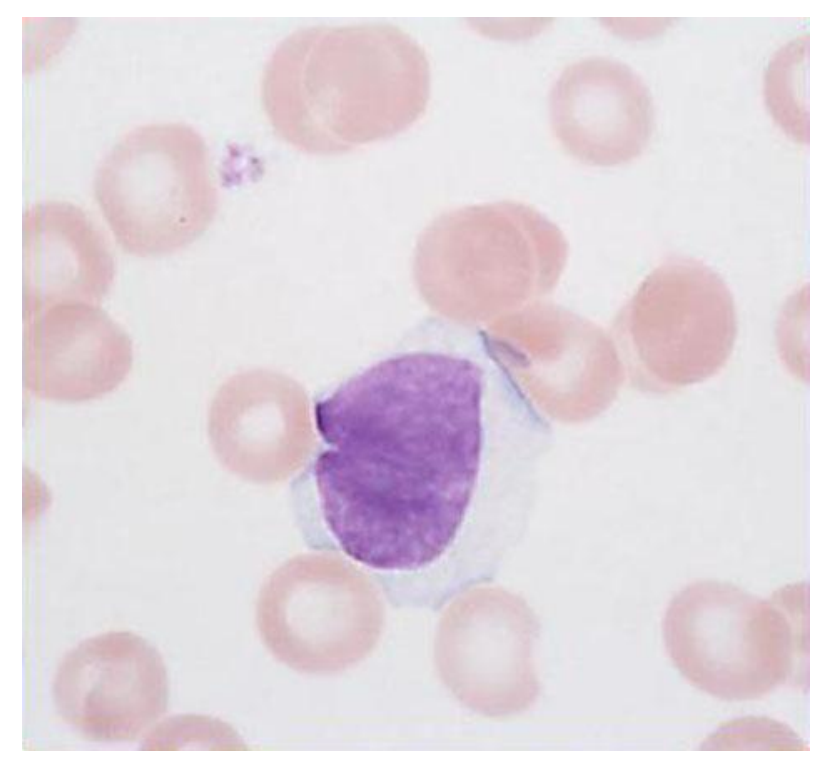

Fig. 3. Atypical lymphocytes (basket cells) were detected in the peripheral blood and bone marrow. No flower cells were observed. 